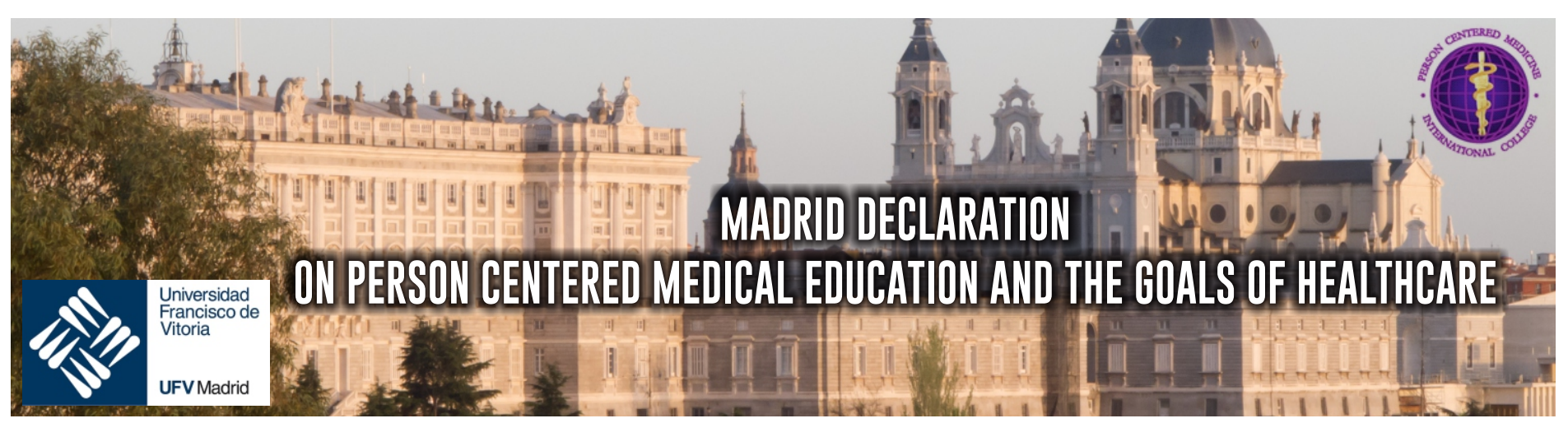

Emerging from the Fourth International Congress of Person Centered Medicine in Madrid on 8 November 2016 and released by the Board of the International College of Person Centered Medicine on 30 June 2017

\title{
Preamble
}

As participants in the Fourth International Congress of Person-Centered Medicine in Madrid, we recognize the compelling need to promote full health for all by transforming in a person-centered manner the way health-care professionals are educated. The current declaration builds on the work of previous conferences and congresses on person centered medicine, particularly those addressed to advance health professional education centered on the whole person.

Healthcare systems are the key resource to achieve improvement in each nation's health and wellbeing . Medical practice is central to the provision of services that enable a continuum of health promotion, disease prevention, diagnosis, disease management, rehabilitation and palliative care services throughout the life course. The practice of medicine in all cultures and societies is currently facing unprecedented challenges in the growing disparities that exist between the legitimate needs of the population and the resources available. Inequity in health and health services within and between countries is proving to be a crucial barrier to well-being around the world.

The 2014 Geneva Declaration on Person- and People-centered Integrated Health Care for All insisted that universal health and well-being require integration of health care planning and delivery at several levels. Such integrated health care must be fully people- and person-centered in order to function in accord with the basic principles of intrinsic human dignity and related values of equity and justice. The World Health Organization is advocating a fundamental shift in the way health services are funded, managed and delivered through an integrated people centered paradigm. The International College of Person Centered Medicine (ICPCM)'s Declaration of Zagreb in 2013 called for action to promote personcentered health for all through transformative educational concepts, programs and actions.

Impressive scientific advances in modern biomedicine have given rise to increasingly narrower medical subspecialties. Clinical care has become depersonalized and fragmented within an increasing and expensive bureaucracy. The growing influence of market forces to transform healthcare systems is changing the ethical basis of medical practice and inducing doctors to abandon their primary commitment to safeguard their patients' wellbeing. The hyperbolic emphasis on science and technology in medical care tends to distance doctors from giving care rooted in genuinely human encounters resulting in doctors' growing dissatisfaction with their professional role with a loss of meaning in their work life.

Against the background of a changeable and complex environment medical education from undergraduate, postgraduate and continuing education needs to help prepare and sustain doctors in the development and nourishment of their professional practice founded on the core values that has underpinned medical practice over the millennia. Critical thinking that is able to confront the increasing dehumanization in medical services must be encouraged and a more comprehensive profile of compassion and competencies adapted to the needs of patients in the 21 st century needs to be fostered among doctors. 
The development of undergraduate medicine training curriculums must comprehensively cover the entire competency dimensions required for person-centred medicine. Together with the scientific knowledge and cognitive (clinical reasoning and decision-making) and psychomotor (manuals and instruments) skills required in clinical work, students must acquire other social, communication and relationship skills and professional attitudes that are essential in rehumanizing clinical practice.

Medical education must include learning methods that foster the spirit of enquiry, discovery, and collaboration to work in interdisciplinary teams Effective mentor guidance and training in real-life situations is essential to enable students to choose, acquire and consolidate important scientific information from clinical experience medical bibliography and online databases. Practicing communication and relationship skills with the patient is an imperative educational aim and an indispensable element of person-centred clinical practice, with proven impact on health outcomes.

The liberal arts (philosophy, literature, history, fine arts, etc.) may serve to bolster personal and humanistic experiences, which increase psychological and cultural sensitivity which will achieve a more comprehensive, empathic and respectful approach to the inner world of patients, helping them to sidestep feelings of isolation and despair during illness.

\section{Call for Action}

Therefore, we propose the following steps to be taken in the education of health care professionals.

1. Universities should assume the responsibility of making a positive and fair selection of their medical students from those candidates who show the best attitude and qualities for personcentered practice.

2. It is advisable to include early programmed clinical placements in degree studies, as they are effective in promoting the empathetic ability of students.

3. Person-centred clinical education and practice must go beyond the limitations of evidence-based medicine and towards one of holistic evidence-informed medicine, enriched by the consideration of experience and values for clinical decision-making.

4. Medical graduates should be able to resolve problems of growing complexity through basic clinical reasoning and prudent decision-making skills in uncertain situations, in which consideration of patient expectations and preferences is essential as well as attention to the values and preferences of the patient and of his or her family.

5. Primary healthcare services represent an ideal environment for this early clinical experience due to the inclusive and continuous nature of care and the establishment of prolonged clinical relationships between patients and their health carers.

6. Educational centres must have available the infrastructure and expert personnel in different technical simulations (robotic, virtual reality, experimental surgery, etc.) that provide technical support to clinical educators.

7. All universities and education providers should have access to expert medical education units, which give support to programs, train clinical teaching personnel and assess the quality of the work accomplished.

8. Person-centred medical education must include in its contents some broad criteria for assessing the outcomes of medical interventions such as clinical effectiveness, safety and costs and the patient's perspective including patient reported outcome measures (PROMs) and patient reported experience measures (PREMs).

9. The accreditation and recertification of practicing doctors must be based on high-quality and wideranging lifelong learning activities.

10. Research must be conducted on the most appropriate methodologies in the humanistic disciplines (anthropology, ethics, human rights, literature, history, deontology and healthcare legislation, pedagogy, the arts, etc), so that such subject areas are meaningfully, acceptably and beneficially taught to students.

The ICPCM calls on educational institutions, associations of health professionals, medical colleges, and governmental and inter-governmental organizations to collaborate in improving health systems across the world, by centering them on the person through medical education programs in graduate, postgraduate and continuing medical education activities. 